Acta Crystallographica Section B

Structural Science,

Crystal Engineering

and Materials

ISSN 2052-5206

\title{
Bis(n-dodecylammonium) tetrachlorozincate
}

M. R. Ciajolo, P. Corradini and V. Pavone

This electronic document was scanned from an archival copy of material deposited to accompany a paper published in an IUCr journal. In many cases the only accessible copy was a microfilm of a poor-quality original. 
a $\quad 32178$

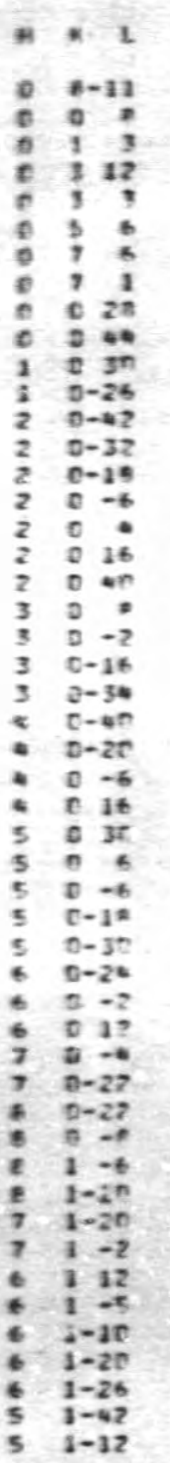

\begin{tabular}{|c|c|c|c|}
\hline Fo & FC & 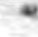 & $\times 1$ \\
\hline 268 & -253 & & $8-10$ \\
\hline 392 & $\cos$ & ? & $c 10$ \\
\hline 278 & -202 & & \\
\hline 536 & -661 & 0 & \\
\hline 1052 & $-04 t$ & & 32 \\
\hline 155 & -120 & ? & 55 \\
\hline 418 & $35 \pi$ & & \\
\hline 360 & 373 & $\pi$ & (1) 18 \\
\hline 177 & 120 & 5 & o 32 \\
\hline 150 & -121 & & $0 \div 0$ \\
\hline 591 & -530 & 1 & \\
\hline 239 & 230 & & $0-26$ \\
\hline 135 & 125 & & $0-4 n$ \\
\hline ספר & 305 & & $x-38$ \\
\hline 601 & -6.59 & 2 & $0-16$ \\
\hline 254 & $-76 \varepsilon$ & $z$ & $x-4$ \\
\hline $1012-$ & 1063 & & $0 \div$ \\
\hline 531 & 526 & 2 & 026 \\
\hline 132 & 135 & & 016 \\
\hline 611 & -622 & 3 & 06 \\
\hline 477 & $\rightarrow 90$ & 3 & \\
\hline 924 & 053 & 3 & $D-18$ \\
\hline 165 & -167 & 3 & $0-38$ \\
\hline 201 & $-1 \times 1$ & . & $0-30$ \\
\hline 96 & 96 & 4 & $9-36$ \\
\hline 1523 & 1561 & 4 & $0 \rightarrow$ \\
\hline 106 & -156 & - & I) 30 \\
\hline 258 & -221 & s & 028 \\
\hline 211 & 225 & 5 & 0 \\
\hline 200 & 2st & & \\
\hline 625 & $-61 \%$ & 5 & $0-20$ \\
\hline 150 & 137 & 5 & $0-32$ \\
\hline 228 & $-22^{\circ}$ & 8 & $0-22$ \\
\hline 279 & -270 & 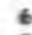 & \\
\hline 323 & 200 & 7 & कह \\
\hline 201 & -187 & $?$ & $0-6$ \\
\hline $3 n 5$ & $2 \pi 0$ & & $9-24$ \\
\hline 191 & -111 & 8 & $0-16$ \\
\hline 200 & 100 & & $0-6$ \\
\hline 261 & 206 & 8 & 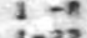 \\
\hline $2 \pi$ & -201 & 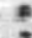 & $1-22$ \\
\hline 211 & 2007 & 7 & $1-3 n$ \\
\hline 175 & 163 & 7 & 1 \\
\hline 421 & -375 & 8 & 120 \\
\hline 266 & 267 & & ; -6 \\
\hline 178 & -175 & 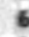 & $1-13$ \\
\hline 345 & 322 & $t$ & $1-21$ \\
\hline 310 & 296 & 6 & $1-23$ \\
\hline 260 & 241 & 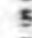 & $1-\infty n$ \\
\hline & & & \\
\hline
\end{tabular}

Fo fo

$163 \quad 145$

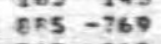

$\begin{array}{rr}217 & 212 \\ 355 & -355\end{array}$

$514+32$
$473-469$

$\begin{array}{rr}473 & -460 \\ 220 & 225\end{array}$

$1237-1236$

$105-160$

$217-201$

$222 \quad 210$

320319

$\begin{array}{ll}106 & 163 \\ 705 & -025\end{array}$

$\begin{array}{rr}829 & -964 \\ 358 & 351\end{array}$

$360-300$

$\begin{array}{ll}660 & -635 \\ 200 & -291\end{array}$

$\begin{array}{ll}975 & 93\end{array}$

$229-236$

in -858

19051086

$119-112$

$307-177$

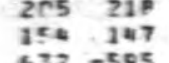

$632-505$

$\begin{array}{lll}158 & 165 \\ 636 & -621\end{array}$

$310-208$

$\begin{array}{ll}167 & -157 \\ 220 & -216\end{array}$

311280

$\begin{array}{lll}1487 & 131 \\ 102 & 183\end{array}$

177
275
2765
-260

${ }_{123}^{275}-260$

138 119

$166-155$

180167

$\begin{array}{lll}361 & -363 \\ 200 & -270 & -20\end{array}$

228.
2200
280

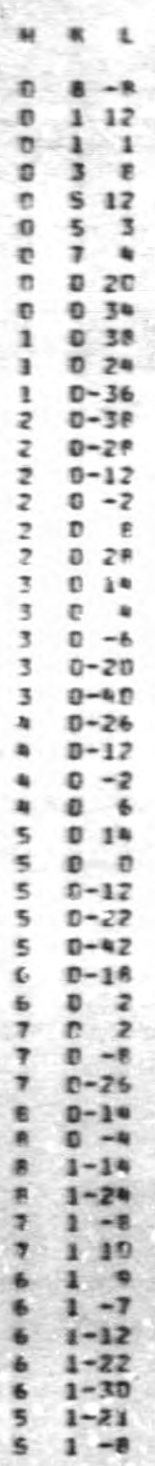

\section{$32178 *$}

$H \times 2$ Fo FC $\mathrm{FC} \times \mathrm{L}$ Fo $\mathrm{FC}$

i. $-1-6 \quad 161200$

$125-101$
$220-235$

3 ins 273

$35 .-330$

$220-206$

$\begin{array}{rr}311 & 358 \\ 305 & -307\end{array}$

$1 \mathrm{az}-162$

$\begin{array}{rrr}100 & -115 \\ 632 & 623\end{array}$

$\begin{array}{rrr}166 & -175 \\ 520 & 529 & 0\end{array}$

$\begin{array}{ll}106 & 179 \\ 520 & -237\end{array}$

-0. -537

$\begin{array}{ll}776 & 786 \\ 045 & 221\end{array}$

$\begin{array}{lll}231 & 255\end{array}$

$\begin{array}{rr}-22 & -617 \\ 8 T 6 & -823\end{array}$

255286

1219.151

$147-146$

$\begin{array}{rl}353 & -330 \\ 716 & 700\end{array}$

10891110

$246 \quad 231$

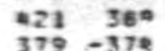

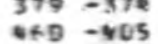

$135-123$

$221-208$
-250

$\begin{array}{ll}250 & -230 \\ 250 & -736\end{array}$

$\begin{array}{ll}228 & -216 \\ 127 & -123\end{array}$

$\begin{array}{ll}127 & -133 \\ 250 & 267\end{array}$

208908

$16 \mathrm{~s}-165$

$\begin{array}{ll}150 & -163 \\ 130 & 130\end{array}$

105162

$226-230$

$167-160$

$160-165$

$\begin{array}{lll}128 & 107 \\ 120 & -109\end{array}$

165148

$\begin{array}{rr}232 & 210 \\ 456 & -943\end{array}$
$: \quad 1: 13223070$

$35-363-303$

- 51 1 $25-413$

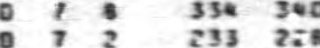

D $26 \quad 10725$

$\begin{array}{lll}0.02 & 202-220 \\ 0 & 312-570\end{array}$

०. 151136

$\begin{array}{lll}0-34 & 125 & 106 \\ 006 & 106\end{array}$

$\begin{array}{lll}0-20 & 357 & 322\end{array}$

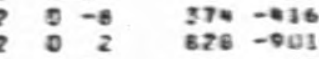

676675

c $10 \quad 360-505$

0 D tEa -706

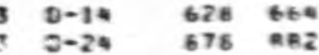

- D-48 $156-128$

$\begin{array}{lll}0-22 & 531 & -532\end{array}$

- 010251066

$\begin{array}{llll}014 & 729-727\end{array}$

$\begin{array}{llll}5 & 0 & 270\end{array}$

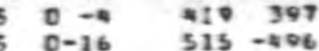

$\begin{array}{lll}0-16 & 315 & -406 \\ 0-26 & 168-150\end{array}$

$\begin{array}{lll}0-42 & 206 & 200\end{array}$

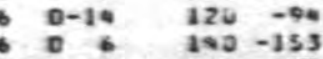

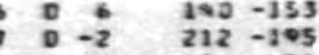

$0-20$ 154 251

- $0-10$ jos 105

$\begin{array}{lll}0-10 & 100 & 105 \\ 1-18 & 230\end{array}$

- $1-18 \quad 220-202$

$71-24 \quad 265173$

? i $\overrightarrow{16} \quad 2110-220$

$60^{6}: \quad 127-113$

ind

5 1-46 $242 \quad 237$

$\begin{array}{llll}5 & 1-10 & 100 & -57 \\ 5 & 1 & 550 & -360\end{array}$

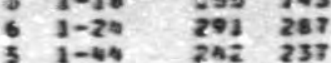

i $i j-20$

$456-45$

$\begin{array}{ll}232 & 216 \\ 278 & 217 \\ 387 & 272\end{array}$ 


\begin{tabular}{|c|c|c|c|c|c|c|c|c|c|c|c|c|c|c|c|c|c|c|c|}
\hline 7 & $\times 2$ & Fo & Ft & N & $\times 2$ & Fo & FC & H & $\times 2$ & 10 & FC & H & $\times 2$ & 50 & $F C$ & n & * & Fo & $F C$ \\
\hline 5 & $1-2$ & 536 & -501 & $\mathbf{s}$ & 10 & 403 & -339 & 5 & 21 & 131 & -131 & 5 & 12 & 352 & -330 & 5 & 2 & 155 & -153 \\
\hline 5 & $1=$ & 200 & -291 & 5 & 15 & 132 & $-1 \times 8$ & 5 & 16 & 199 & -190 & 5 & 113 & 182 & -185 & 5 & 220 & 524 & 496 \\
\hline 5 & $12 ?$ & 311 & $30 r$ & 5 & 120 & 233 & 233 & 5 & 126 & 135 & 136 & $:$ & 134 & 152 & -160 & * & 132 & 150 & -160 \\
\hline$\bullet$ & 122 & 164 & 165 & $*$ & $12 \pi$ & 178 & 173 & 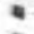 & 115 & 185 & -193 & $\bullet$ & 116 & 414 & 403 & $*$ & 123 & 217 & 218 \\
\hline$:$ & 110 & 245 & 153 & : & 18 & 208 & 277 & $*$ & 17 & 156 & -167 & $:$ & 16 & 460 & 440 & $:$ & 15 & 127 & -119 \\
\hline$:$ & 15 & 639 & 598 & $*$ & 12 & 769 & 781 & 4 & 10 & 810 & 763 & $\bullet$ & $1-2$ & 361 & 338 & 4 & $2-3$ & 312 & -298 \\
\hline$\bullet$ & $1-4$ & $7>0$ & 137 & - & $1-5$ & 207 & 215 & 4 & $1-6$ & 224 & 254 & $*$ & $2-8$ & 158 & -133 & 4 & $1-10$ & 406 & -401 \\
\hline 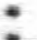 & $1-12$ & 636 & $-6+6$ & $*$ & $1-14$ & E11 & -777 & 4 & $1-16$ & 877 & -880 & $*$ & $1-18$ & 771 & -745 & $\bullet$ & $1-19$ & 437 & 433 \\
\hline$\vdots$ & $1-20$ & 556 & -537 & : & $\mid-21$ & 372 & -340 & $*$ & $1-22$ & 358 & -357 & $\because$ & $1-23$ & ins & -117 & $:$ & $1-20$ & 262 & -252 \\
\hline 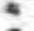 & $1-26$ & 270 & -151 & 3 & $2-43$ & 142 & -143 & 3 & $1-42$ & $1 \times 3$ & -199 & 3 & $1-41$ & 263 & -162 & 3 & $1-0$ & 332 & -304 \\
\hline 3 & $3-30$ & 163 & -171 & 3 & $1-3 n$ & 405 & -461 & 3 & $1-37$ & 201 & -212 & 3 & $1-20$ & 169 & 156 & 3 & $1-28$ & $20 \mathrm{a}$ & -216 \\
\hline 3 & $:-2 ?$ & 235 & 212 & 3 & $1-25$ & 205 & 315 & 3 & $1-23$ & 323 & 201 & 3 & $1-22$ & 123 & 122 & 3 & $1-21$ & 250 & 242 \\
\hline $\begin{array}{l}3 \\
3\end{array}$ & $1-20$ & 90 & 116 & 3 & $1-10$ & 426 & -401 & 3 & $1-17$ & 172 & -150 & 3 & $1-16$ & 129 & 139 & 3 & $2-15$ & 259 & -247 \\
\hline 3 & $1-10$ & 263 & 270 & 3 & $1-13$ & $30 s$ & -303 & 3 & $1-12$ & 329 & 342 & 3 & $1-11$ & 203 & -295 & 3 & $1-10$ & 360 & 365 \\
\hline 7 & 10 & 215 & -235 & 3 & $1 \rightarrow$ & 438 & 066 & 3 & $1-7$ & 135 & -130 & 3 & $1-b$ & 597 & 567 & 3 & $1-5$ & 145 & -132 \\
\hline 3 & $1-4$ & $5 x c$ & 835 & 3 & $1-3$ & 134 & $12 \mathrm{~b}$ & 3 & $1-2$ & 654 & 605 & 3 & $1-1$ & 266 & -242 & 3 & 2 & 1155 & 1155 \\
\hline 3 & 12 & 9045 & $\bullet 26$ & 3 & 3 & 111 & $10 s$ & 3 & 4 & $6 * 0$ & 578 & 3 & 15 & $2 \pi 2$ & 190 & 3 & 1 & 207 & 292 \\
\hline 3 & 12 & 220 & 224 & 3 & $\circ$ & $1<9$ & 153 & 3 & 120 & 207 & -300 & 3 & 112 & $4 c 0$ & -364 & 3 & 114 & 305 & -312 \\
\hline 3 & $11^{5}$ & $7<6$ & 702 & 3 & 1216 & 936 & -907 & 3 & 131 & 161 & -165 & 3 & 132 & 230 & -240 & 3 & 134 & 126 & -131 \\
\hline 3 & $23 z$ & 204 & -207 & 3 & 128 & 192 & -217 & 2 & 142 & 104 & 127 & 2 & 140 & 172 & 188 & 2 & 138 & 233 & 254 \\
\hline 2 & 156 & 222 & 201 & 2 & 130 & 401 & 420 & 2 & 132 & 1 (A2 & 192 & 2 & 130 & 274 & 277 & 2 & 126 & 129 & -135 \\
\hline 7 & 120 & *0.5 & -608 & 2 & 122 & 624 & -601 & 2 & 120 & 727 & -742 & 2 & 118 & 815 & -662 & 2 & 116 & $1525-$ & -1529 \\
\hline 2 & 135 & 925 & -017 & 2 & 110 & 711 & ירסיר & 2 & 113 & 450 & -476 & 2 & 112 & 574 & -576 & 2 & 111 & 396 & -370 \\
\hline 2 & $11^{n}$ & 196 & -105 & 2 & 1 & 393 & -178 & 2 & 1 & 214 & 181 & 2 & 17 & 275 & -275 & 2 & 1 & 503 & 470 \\
\hline 2 & $\bullet$ & 496 & 561 & 2 & 1 & 330 & 341 & 2 & 1 & 642 & 638 & 2 & 1 & 560 & 602 & 2 & 1 & 734 & 696 \\
\hline 2 & $3-2$ & $15 a$ b & $25+7$ & 2 & $1-2$ & 795 & -844 & 2 & $1-3$ & 587 & 581 & 2 & 20 & 561 & -559 & 2 & $1-5$ & 961 & 921 \\
\hline 2 & $2-6$ & TRE & -743 & 2 & $1-7$ & 605 & 6et & 2 & $1-x$ & 869 & -268 & 2 & $1 \rightarrow 9$ & 220 & 245 & 2 & $2-10$ & 559 & -569 \\
\hline 2 & $a-12$ & $2 n$ & -205 & 2 & $1-13$ & srs & -405 & $?$ & $1-10$ & 403 & .00 & 2 & $1-15$ & 724 & -716 & 2 & $1-16$ & 633 & 635 \\
\hline 2 & $|-1\rangle$ & $2270-$ & $-1 \geq 56$ & 2 & $1-10$ & $11 n 2$ & 1090 & 2 & $1-20$ & 1005 & 1037 & 2 & $1-21$ & .03 & -387 & 2 & $1-33$ & 226 & 239 \\
\hline 2 & $2-30$ & 327 & -938 & 2 & $x-3 s$ & $3 \in 7$ & -335 & 2 & $1-40$ & 347 & -322 & 2 & $1-42$ & 228 & -225 & 1 & $1-40$ & 302 & 310 \\
\hline 2 & $1-42$ & 400 & 406 & 1 & $1-40$ & 430 & 436 & 1 & $1-36$ & 475 & $4+6$ & 1 & $1-36$ & 500 & 543 & 1 & $1-30$ & 224 & -216 \\
\hline 2 & $2-32$ & 193 & -175 & 2 & $1-31$ & 276 & -243 & 1 & $1-20$ & 235 & -200 & 1 & $1-28$ & 196 & -174 & 1 & $1-12$ & 296 & 267 \\
\hline 2 & $t-13$ & 316 & 93 & 1 & $1-1 n$ & 307 & -412 & 1 & $1=0$ & 126 & 126 & 1 & & 952 & -1050 & 1 & & $1152-$ & -1250 \\
\hline 1 & $c \rightarrow$ & 273 & -273 & 1 & $1-4$ & $1017-$ & -1103 & 1 & $1-3$ & 263 & -183 & 2 & $1-2$ & 9540 & -1031 & 1 & $1-2$ & 150 & -146 \\
\hline 1 & $2 n$ & 566 & -630 & 1 & 1 & 527 & 535 & 1 & 1 & 285 & -158 & 1 & 16 & $10>0-$ & -1062 & 1 & 13 & 549 & -536 \\
\hline$\frac{3}{1}$ & 15 & 605 & -622 & 1 & 28 & 310 & -673 & I & 110 & 281 & -290 & 1 & 111 & 538 & -526 & 1 & 112 & 125 & -130 \\
\hline 3 & 122 & 572 & $5=0$ & 1 & 123 & 303 & 347 & 1 & 120 & 502 & 498 & 1 & 125 & 305 & 362 & 1 & 126 & 502 & 497 \\
\hline 1 & 227 & 206 & 253 & 3 & 128 & 468 & 480 & 1 & 130 & 331 & 340 & 1 & 231 & 131 & -149 & 1 & 134 & 450 & 498 \\
\hline$\frac{1}{0}$ & 135 & 201 & -236 & 1 & 136 & 113 & 149 & 0 & 140 & 231 & -237 & D & 138 & 329 & -304 & D & 236 & 413 & -396 \\
\hline ह & 130 & 200 & 101 & 0 & 232 & 225 & -211 & D & 128 & 183 & 168 & 0 & 126 & 230 & 232 & 0 & 224 & 254 & 202 \\
\hline e & 122 & 313 & 270 & $n$ & 120 & 525 & 490 & 0 & 219 & $1 * 3$ & 163 & 0 & 118 & 950 & \$27 & 0 & 116 & 687 & 622 \\
\hline c & 114 & 807 & 120 & 0 & $2-42$ & 210 & 185 & D & $2-40$ & 333 & 284 & D & $2-38$ & 466 & 386 & 0 & $2-36$ & 623 & 497 \\
\hline c & $2-35$ & 276 & $-2 c z$ & $n$ & $2-30$ & $1<9$ & -170 & 0 & $2-33$ & 210 & -105 & $c$ & 232 & 5.63 & 602 & 0 & $2-30$ & 435 & 432 \\
\hline 0 & $2-27$ & 106 & 100 & 0 & $2-27$ & 121 & 100 & D & $2-25$ & 250 & 257 & 0 & $2-23$ & 312 & 305 & 0 & $z-22$ & 218 & 232 \\
\hline 5 & $2-21$ & 135 & 108 & $n$ & $2-20$ & 221 & 212 & 0 & $2-17$ & 1107 & 1171 & 0 & $2-16$ & 155 & 154 & 0 & $2-14$ & 248 & 231 \\
\hline c & $2-22$ & 201 & 220 & 1 & 2.42 & 172 & 201 & 2 & 241 & 260 & -154 & 1 & 240 & 258 & 284 & 1 & 239 & 146 & -141 \\
\hline 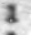 & 236 & 345 & 363 & 1 & $=36$ & 432 & 45 & 1 & 230 & 670 & 125 & 1 & 233 & 228 & 248 & 1 & 232 & 186 & 188 \\
\hline 1 & 231 & 122 & 200 & 1 & 230 & 131 & 134 & -1 & 229 & 367 & 170 & 1 & 228 & 241 & -136 & 1 & 227 & 210 & 197 \\
\hline 1 & 226 & 502 & -404 & 2 & 224 & 830 & -790 & 3 & 222 & 1n71- & $-1 \leq 08$ & 1 & 221 & 116 & -121 & 1 & 220 & $1184-$ & 1100 \\
\hline 1 & 210 & 210 & -215 & 1 & 2 & 100 & -102 & 1 & 28 & 309 & 269 & 1 & 26 & 610 & 523 & 1 & 2 & 270 & 273 \\
\hline
\end{tabular}




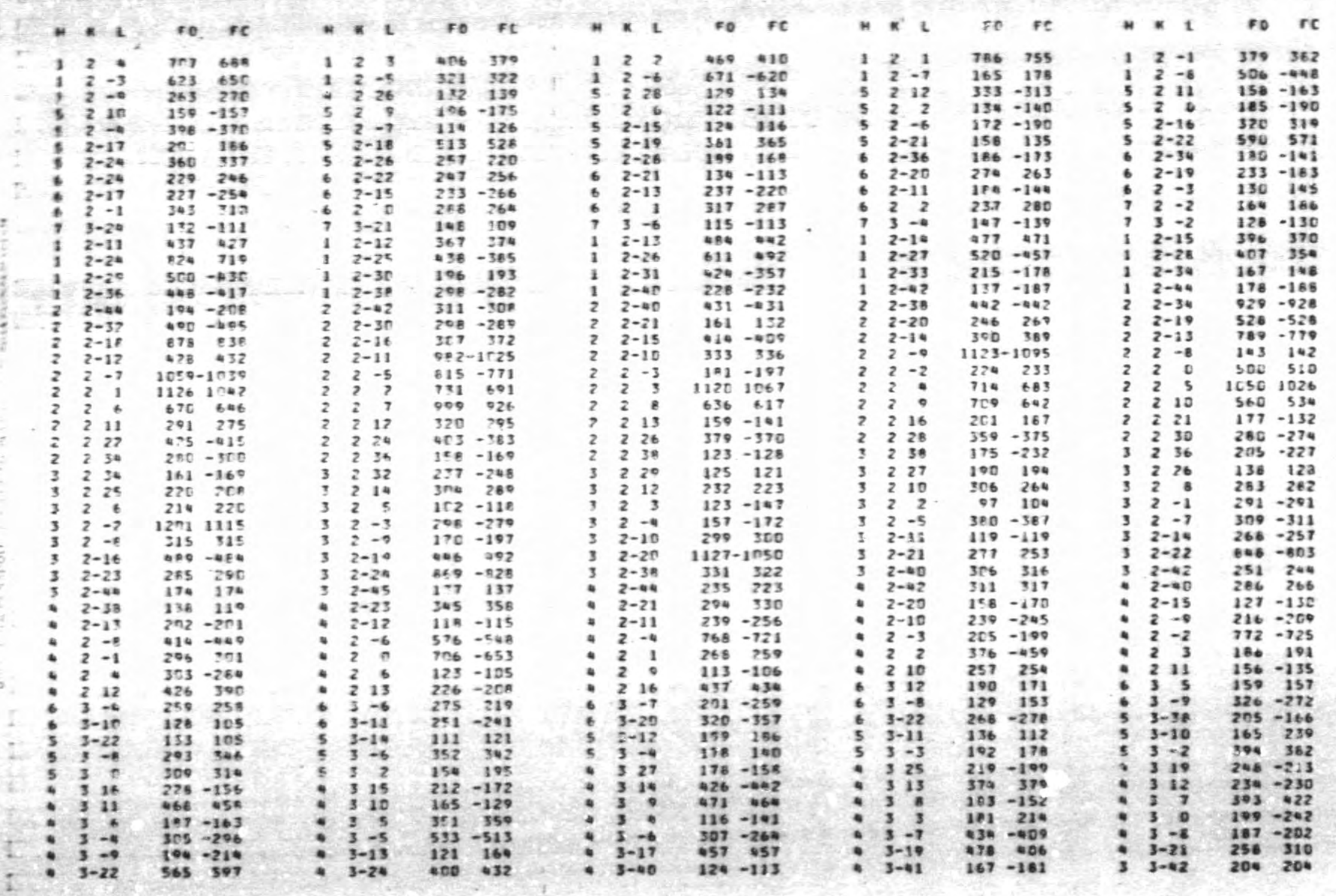




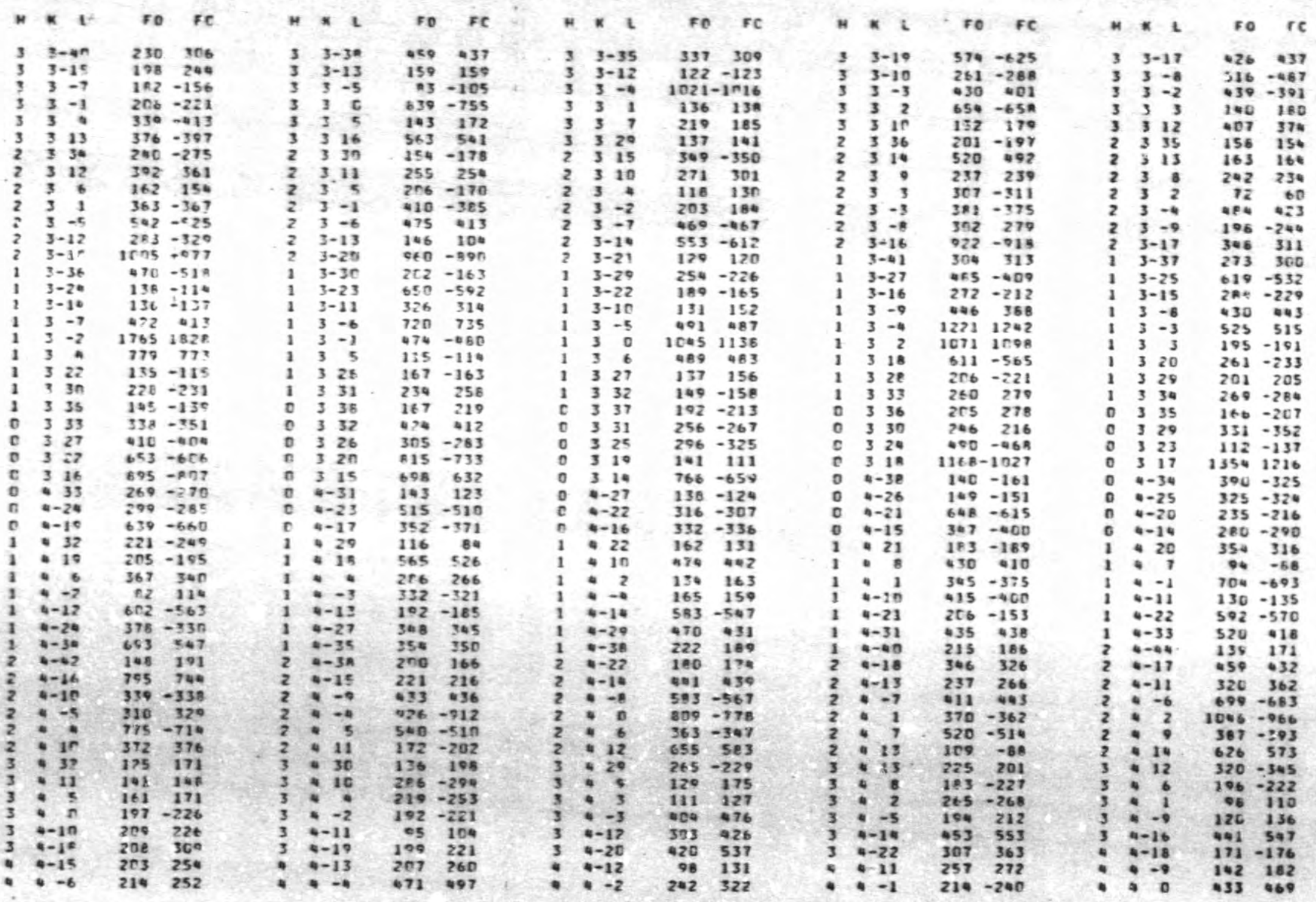




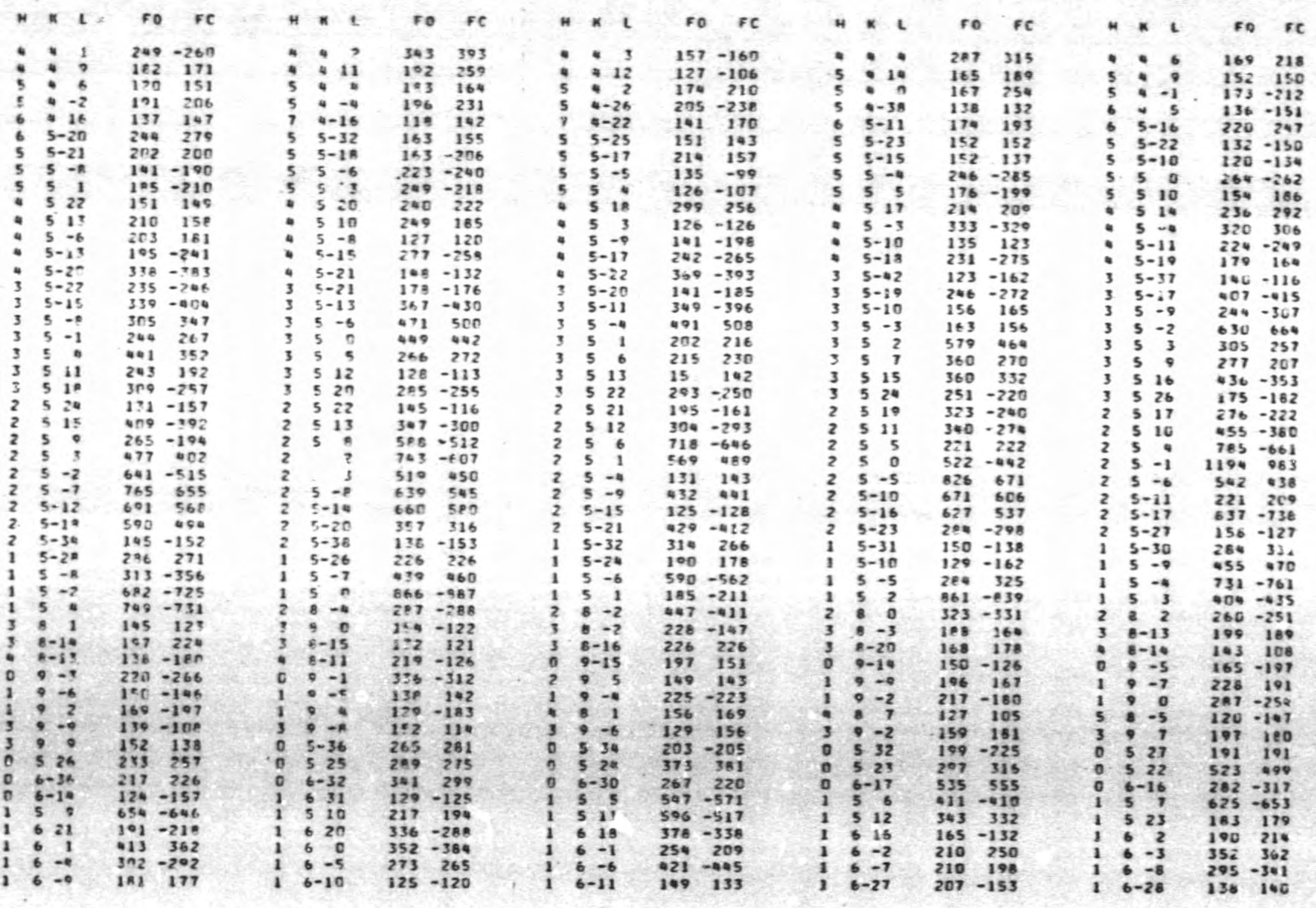




\begin{tabular}{|c|c|c|c|c|c|c|c|c|c|c|c|c|c|c|c|c|c|c|c|}
\hline w & $\approx 2$ & 50 & FC & H & $\times 2$ & ro & FC & $H$ & K L & 50 & FC & $\omega$ & $\pi \mathrm{l}$ & Fo & FC & N & $\times 2$ & 10 & fC \\
\hline$\frac{1}{2}$ & $6-31$ & 157 & -130 & 1 & $6-32$ & 161 & -140 & 1 & $6-36$ & 277 & -256 & 1 & $6-35$ & 103 & -210 & 1 & $\begin{array}{l}6-40 \\
6-19\end{array}$ & $\begin{array}{l}156 \\
273\end{array}$ & $\begin{array}{l}-188 \\
-3146\end{array}$ \\
\hline 2 & $\begin{array}{l}6-42 \\
6-16\end{array}$ & $\begin{array}{c}129 \\
399\end{array}$ & $\begin{array}{l}-127 \\
-391\end{array}$ & ${ }_{2}^{2}$ & $\begin{array}{l}6-38 \\
6-15\end{array}$ & $\begin{array}{l}203 \\
176\end{array}$ & $\begin{array}{l}-227 \\
-204\end{array}$ & 2 & $\begin{array}{l}6-36 \\
6-16\end{array}$ & $\begin{array}{l}219 \\
239\end{array}$ & $\begin{array}{l}-220 \\
-279\end{array}$ & 2 & $\begin{array}{l}6-20 \\
6-13\end{array}$ & $\begin{array}{l}163 \\
283\end{array}$ & $\begin{array}{l}-157 \\
-333\end{array}$ & 2 & $\begin{array}{l}6-19 \\
6-11\end{array}$ & $\begin{array}{l}273 \\
276\end{array}$ & $\begin{array}{l}-366 \\
-326\end{array}$ \\
\hline 2 & $6-0$ & $2 \geq 7$ & -257 & 2 & $6-9$ & 296 & 350 & 2 & $6-7$ & 263. & -130 & 2 & $6-6$ & 687 & 503 & 2 & $6-3$ & 195 & $\begin{array}{l}-326 \\
-175\end{array}$ \\
\hline 2 & $6=0$ & 537 & 575 & 2 & $6-3$ & 101 & -153 & 2 & $6-2$ & 608 & 670 & 2 & $6-1$ & 108 & 205 & 2 & 0 & 538 & 630 \\
\hline$\frac{2}{2}$ & & $\begin{array}{l}103 \\
2 \times 5 .\end{array}$ & 168 & 2 & 6. 2 & $\begin{array}{r}435 \\
265\end{array}$ & 470 & 2 & 63 & 227 & 244 & 2 & & 255 & 265 & 2 & 65 & $\begin{array}{l}273 \\
351\end{array}$ & $\begin{array}{r}239 \\
-290\end{array}$ \\
\hline 3 & 83 & 150 & -194 & 3 & & 196 & $\begin{array}{r}166 \\
-196\end{array}$ & 3 & $b-1$ & $\begin{array}{l}122 \\
230\end{array}$ & $\begin{array}{r}123 \\
-216\end{array}$ & 3 & $\begin{array}{ll}6 & 22 \\
6 & -2\end{array}$ & $\begin{array}{l}152 \\
224\end{array}$ & 213 & 3 & $6-3$ & 236 & $\begin{array}{l}-104 \\
-190\end{array}$ \\
\hline 3 & $6-5$ & 263 & -253 & 3 & $5-6$ & 157 & 173 & 3 & $6-7$ & 245 & -228 & 3 & $6-8$ & 219 & 213 & 3 & $6-9$ & $15 z$ & -197 \\
\hline 3 & $6-11$ & 203 & -155 & 3 & $6-14$ & 276 & -307 & 3 & $6-16$ & 350 & -396 & 3 & $6-17$ & 134 & -132 & 3 & $6-18$ & 227 & -220 \\
\hline 3 & $6-2 c$ & 240 & -253 & 3 & $6-22$ & 205 & -213 & 5 & $6-34$ & 213 & 125 & 4 & $6-24$ & 201 & -175 & 4 & $6-23$ & 159 & 107 \\
\hline 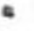 & $6-27$ & 210 & -215 & 4 & $6-21$ & 152 & 83 & 4 & $6-18$ & 164 & -156 & 4 & $6-17$ & 100 & 167 & 4 & $6-16$ & 176 & -153 \\
\hline 4 & $6-15$ & 253 & 219 & 4 & $6-14$ & ${ }^{\circ} 136$ & -135 & 4 & $6-13$ & 170 & 127 & 4 & $6-12$ & 145 & -110 & 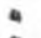 & $6-3$ & 218 & $\begin{array}{l}-211 \\
-235\end{array}$ \\
\hline$:$ & $\$ 1$ & 201 & $-1 \ell 2$ & $\cdot$ & 63 & 259 & -196 & 4 & & 159 & -190 & $\therefore$ & & $\begin{array}{l}249 \\
178\end{array}$ & $\begin{array}{l}-212 \\
-132\end{array}$ & 4 & $6 \quad 6$ & $\begin{array}{l}201 \\
205\end{array}$ & $\begin{array}{r}-235 \\
165\end{array}$ \\
\hline 5 & $\begin{array}{l}6 \\
t \\
t\end{array}$ & 178 & -191 & 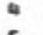 & 68 & 191 & -221 & $:$ & 610 & $\begin{array}{l}133 \\
118\end{array}$ & $\begin{array}{l}-130 \\
-13 n\end{array}$ & 5 & $\begin{array}{ll}6 & 11 \\
6 & -3\end{array}$ & 140 & $\begin{array}{r}-132 \\
152\end{array}$ & 5 & $\begin{array}{ll}6 & 16 \\
6 & -5\end{array}$ & 134 & 132 \\
\hline$\epsilon$ & $\begin{array}{lr}6 & 1 \\
6 & -6\end{array}$ & $\begin{array}{l}163 \\
172\end{array}$ & $\begin{array}{l}126 \\
233\end{array}$ & $\begin{array}{l}5 \\
6\end{array}$ & $\begin{array}{ll}6 & -1 \\
6 & -4\end{array}$ & $\begin{array}{l}129 \\
116\end{array}$ & $\begin{array}{l}158 \\
158\end{array}$ & $\begin{array}{l}5 \\
6\end{array}$ & $\begin{array}{ll}6 & -2 \\
6 & -1\end{array}$ & 126 & 136 & 6 & 63 & 108 & 100 & 6 & & 173 & -111 \\
\hline 6 & 70 & 159 & 132 & 6 & $7-4$ & $1 \geqslant 1$ & 151 & & $7-20$ & 125 & 113 & 5 & $7-3$ & 118 & 131 & 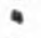 & 718 & 169 & -198 \\
\hline 4 & 716 & $2=4$ & -193 & 4 & & 224 & 193 & 4 & $7-1$ & 170 & 119 & 4 & $7-5$ & 183 & 201 & $:$ & $7-6$ & 123 & -143 \\
\hline 4 & $7-e$ & 192 & 202 & $=$ & $7-10$ & 146 & -156 & 4 & $7-12$ & 122 & -172 & 3 & $7-17$ & 322 & 310 & 3 & $1-4$ & 226 & -239 \\
\hline 3 & $2-3$ & 263 & 127 & 3 & $7-1$ & 167 & -173 & 3 & 72 & 182 & -215 & 3 & 75 & 115 & -111 & 3 & & 136 & -123 \\
\hline 2 & 724 & 158 & 185 & 2 & 722 & 198 & 196 & 2 & 720 & 157 & 166 & 2 & 77 & 188 & -164 & 2 & & 115 & 122 \\
\hline 2 & 75 & $1>0$ & -126 & 2 & & 177 & 132 & 2 & & 131 & 84 & 2 & & 193 & 174 & 2 & $7-1$ & 152 & -104 \\
\hline 2 & $7-5$ & 175 & -140 & 2 & $7-7$ & 111 & -112 & 2 & $7-10$ & 127 & -102 & 2 & $7-11$ & 139 & -218 & 2 & $7-13$ & 225 & -168 \\
\hline 2 & $7-15$ & 264 & -256 & 2 & $7-16$ & 157 & -155 & 2 & $7-17$ & 105 & -151 & 2 & $7-19$ & 237 & -160 & 2 & $7-19$ & $\begin{array}{l}225 \\
155\end{array}$ & -225 \\
\hline 2 & $7-2 n$ & 241 & -233 & 2 & $7-22$ & 170 & -123 & 2 & $7-28$ & 135 & -140 & 2 & $7-3 n$ & 135 & -102 & 1 & $7-30$ & $\begin{array}{l}155 \\
152\end{array}$ & $\begin{array}{l}-200 \\
-100\end{array}$ \\
\hline 1 & $7-32$ & 232 & -220 & 1 & $7-30$ & 250 & -210 & 1 & $7-28$ & 232 & -187 & 1 & $7-25$ & 212. & -151 & 1 & $7-23$ & $\begin{array}{l}152 \\
151\end{array}$ & \\
\hline 1 & $7-22$ & 105 & 157 & 1 & $7-20$ & 162 & 137 & 1 & $7-0$ & 191 & 117 & 1 & $7-2$ & 313 & 312 & 1 & $\begin{array}{ll}7 & -1 \\
7 & 21\end{array}$ & 170 & $\begin{array}{r}-150 \\
215\end{array}$ \\
\hline 1 & 70 & 226 & 217 & 1 & 72 & 251 & 208 & 1 & 7 & 226 & 183 & $\begin{array}{l}1 \\
0\end{array}$ & & $\begin{array}{l}181 \\
163\end{array}$ & $\begin{array}{r}155 \\
-201\end{array}$ & 1 & $\begin{array}{ll}7 & 21 \\
7 & 18\end{array}$ & $\because 97$ & -675 \\
\hline $\begin{array}{l}0 \\
\mathrm{c}\end{array}$ & 720 & 352 & -376 & 0 & 722 & 377 & -406 & 0 & $\begin{array}{l}720 \\
8-22\end{array}$ & 423 & -382 & 0 & $\begin{array}{l}719 \\
8-20\end{array}$ & 224 & -169 & 0 & $8-16$ & & -108 \\
\hline c & $\begin{array}{l}716 \\
8-15\end{array}$ & 360 & -328 & $\begin{array}{l}0 \\
0 \\
0\end{array}$ & $\begin{array}{l}8-24 \\
3-12\end{array}$ & $\begin{array}{l}188 \\
165\end{array}$ & -145 & 0 & $\begin{array}{r}8-22 \\
80\end{array}$ & $\begin{array}{l}226 \\
352\end{array}$ & $\begin{array}{r}-184 \\
327\end{array}$ & 1 & $22^{\circ}$ & 208 & 131 & 1 & 816 & 70 & $150^{\circ}$ \\
\hline 1 & $\begin{array}{l}8-15 \\
815\end{array}$ & $\begin{array}{l}206 \\
252\end{array}$ & $\begin{array}{l}-163 \\
-237\end{array}$ & $\begin{array}{l}0 \\
1\end{array}$ & $\begin{array}{l}5-1 ? \\
89\end{array}$ & $\begin{array}{l}165 \\
176\end{array}$ & $\begin{array}{l}-166 \\
-164\end{array}$ & $\begin{array}{l}0 \\
1\end{array}$ & 87 & 169 & -162 & 1 & & 133 & 141 & 1 & $8-1$ & 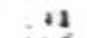 & -135 \\
\hline 1 & e -5 & 100 & $\begin{array}{l}-237 \\
-122\end{array}$ & 1 & $9-7$ & $1 * 1$ & -186 & i & $8-9$ & 181. & -195 & 1 & $8-11$ & 129 & -140 & 1. & $8-14$ & 163 & -197 \\
\hline 1 & $8-1)^{2}$ & 289 & -277 & 1 & $8-17$ & 180 & -559 & 1 & $8-19$ & 147 & 165 & 1 & $8-22$ & 190 & -166 & 2 & $8-27$ & $\begin{array}{r}163 \\
1067\end{array}$ & 151 \\
\hline 2 & $8-5$ & 161 & -192 & 2 & $8-6$ & 240 & -227 & 1 & 018 & 1375 & 1045 & 1 & D 16 & 622 & 651 & 1 & $\begin{array}{lll}0 & 14 \\
0 & 4\end{array}$ & $\begin{array}{c}1067 \\
660\end{array}$ & $\begin{array}{r}1133 \\
123\end{array}$ \\
\hline 1 & 012 & 868 & RBd & 1 & 0.10 & 608 & 576 & 1 & 0 & 655 & 682 & 1 & & 757 & $\begin{array}{r}220 \\
-1370\end{array}$ & 1 & $0-12$ & $1380-$ & $=1567$ \\
\hline 1 & $0 ?$ & 340 & 301 & 1 & $0-6$ & 303 & -421 & 1 & $0-n$ & 1060 & $\begin{array}{c}-044 \\
-11 * 9\end{array}$ & 1 & $\begin{array}{l}0-10 \\
0-20\end{array}$ & $\begin{array}{c}1253- \\
628\end{array}$ & $\begin{array}{r}-1378 \\
-714\end{array}$ & $i$ & $0-22$ & $3=0$ & -345 \\
\hline 1 & $0-1=$ & $1240-$ & -1390 & 1 & $0-16$ & $1035-$ & -1093 & 1 & $0-18$ & 332 & 308 & 2 & $0 \quad 20$ & 338 & 358 & 2 & & 386 & 366 \\
\hline$?$ & $c-26$ & 336 & $36 n$ & 2 & $0-24$ & 270 & 272 & 2 & $\begin{array}{c}018 \\
0-28\end{array}$ & 254 & 253 & 3 & $0-32$ & 105 & -146 & 5 & 020 & 163 & 157 \\
\hline $\begin{array}{l}2 \\
5\end{array}$ & c 24 & 408 & 422 & 3 & $0-26$ & 575 & 576 & 3 & $\begin{array}{l}0-28 \\
0-38\end{array}$ & 104 & 202 & 6 & $0-40$ & 239 & 220 & 6 & $0-38$ & 278 & 277 \\
\hline 6 & $\begin{array}{l}0.18 \\
c-36\end{array}$ & $2 n 2$ & 257 & 5 & C 15 & 222 & $\begin{array}{l}214 \\
196\end{array}$ & $\begin{array}{l}5 \\
6\end{array}$ & $\begin{array}{l}0-36 \\
0.16\end{array}$ & 266 & 278 & 6 & 018 & 203 & 301 & 6 & 020 & 236 & 230 \\
\hline i & $\begin{array}{l}c-3 b \\
1-36\end{array}$ & $\begin{array}{l}223 \\
129\end{array}$ & $\begin{array}{r}227 \\
-189\end{array}$ & 7 & $\begin{array}{l}0-30 \\
1-30\end{array}$ & 179 & $\begin{array}{r}196 \\
-147\end{array}$ & 5 & $1-37$ & 150 & $-15 \theta$ & 5 & 115 & 171 & 273 & 5 & 116 & 310 & 302 \\
\hline 5 & 317 & $\begin{array}{l}18 . \\
152\end{array}$ & $\begin{array}{r}-189 \\
147\end{array}$ & 5 & 118 & 356 & 363 & 5 & 120 & 350 & 363 & 3 & $1-36$ & 505 & -525 & 3 & $1-32$ & 407 & -013 \\
\hline 3 & $1-3 n$ & 308 & -315 & 3 & 118 & 500 & -502 & 3 & $12 n$ & 200 & -265 & 2 & $1-22$ & 1108 & 1121 & 2 & $1-23$ & 266 & -251 \\
\hline 2 & $1-20$ & 933 & 948 & 2 & $1-26$ & 615 & 648 & 2 & $1-28$ & 368 & 356 & 2 & $1-29$ & 205 & 217 & 2 & $\begin{array}{l}1-30 \\
1-20\end{array}$ & $\begin{array}{l}136 \\
451\end{array}$ & 105 \\
\hline 2 & $1-31$ & 232 & 256 & $\begin{array}{l}1 \\
1\end{array}$ & $\begin{array}{l}1-26 \\
1-18\end{array}$ & $\begin{array}{l}128 \\
588\end{array}$ & -109 & 1 & $\begin{array}{l}1-22 \\
1-17\end{array}$ & $\begin{array}{l}282 \\
182\end{array}$ & $\begin{array}{l}276 \\
131\end{array}$ & $\begin{array}{l}1 \\
1\end{array}$ & $\begin{array}{l}1-21 \\
1-16\end{array}$ & $\begin{array}{l}134 \\
651\end{array}$ & $\begin{array}{l}105 \\
641\end{array}$ & 1 & $1-15$ & 390 & $370^{\circ}$ \\
\hline
\end{tabular}




\begin{tabular}{|c|c|c|c|c|c|c|c|c|c|c|c|c|c|c|c|c|c|c|c|}
\hline$n$ & $\times 1$ & 50 & $\mathrm{FC}$ & H & $\times L$ & Fo & FC & $\mu$ & K. 2 & 50 & FC & H & $\times t$ & 10 & IC & N & * & 60 & fC \\
\hline 1 & $1-14$ & $6=3$ & 656 & 1 & $1-13$ & 408 & 402 & 1 & 113 & 118 & -134 & 1 & 115 & 162 & 400 & 1 & 116 & 286 & -263 \\
\hline 1 & $\begin{array}{ll}117 \\
13\end{array}$ & श्र & -702 & 1 & 118 & 677 & 503 & 1 & 119 & 99 & 101 & 1 & 120 & 653 & 627 & 1 & 121 & 310 & $\begin{array}{r}319 \\
-536\end{array}$ \\
\hline$i$ & $\begin{array}{ll}1 & 13 \\
2 & 10\end{array}$ & $\begin{array}{l}154 \\
5 \text { ps }\end{array}$ & $\begin{array}{l}-147 \\
-596\end{array}$ & 1 & $\begin{array}{ll}2 & 19 \\
2 & 13\end{array}$ & $\begin{array}{r}1050- \\
581\end{array}$ & $\begin{array}{r}-1013 \\
-507\end{array}$ & 1 & $\begin{array}{ll}2 & 17 \\
2 & 12\end{array}$ & $\begin{array}{l}273 \\
376\end{array}$ & $\begin{array}{l}-250 \\
-370\end{array}$ & 1 & $\begin{array}{ll}216 \\
211\end{array}$ & $\begin{array}{l}527 \\
630\end{array}$ & $\begin{array}{l}-511 \\
-401\end{array}$ & $!$ & $\begin{array}{ll}2 & 15 \\
2 & 17\end{array}$ & $\begin{array}{l}515 \\
257\end{array}$ & $\begin{array}{l}-536 \\
-267\end{array}$ \\
\hline - & 210 & 257 & 207 & 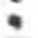 & 210 & 182 & -202 & $:$ & 220 & 201 & 209 & $?$ & 222 & 159 & 160 & $\because$ & 220 & 103 & 100 \\
\hline 5 & 220 & 215 & -222 & 5 & 238 & 319 & -326 & 5 & 216 & 379 & -375 & 5 & $2-29$ & 161 & -163 & 5 & $2-30$ & 136 & 819 \\
\hline 5 & $2-31$ & 179 & -163 & 6 & $2-3 x$ & $2 \in 9$ & -283 & 7 & $2-20$ & 205 & -275 & 7 & $2-23$ & 159 & -160 & 3 & $2-16$ & $3 e 5$ & COs \\
\hline 1 & $2-1=$ & 715 & 767 & 1 & $z-19$ & 106 & 156 & 1 & $2-20$ & 762 & 785 & 1 & $2-21$ & 122 & -141 & 1 & $2-22$ & 931 & 807 \\
\hline 1 & $2-23$ & 262 & -250 & 2 & $2-2^{\circ}$ & 118 & 126 & 2 & $2-27$ & 196 & 190 & 2 & $2-26$ & 125 & -127 & 2 & $2-25$ & 262 & 260 \\
\hline 2 & $2-25$ & 310 & 292 & 2 & $21 \gamma$ & 677 & -833 & 2 & 218 & 436 & -238 & 2 & 219 & 360 & -352 & 2 & 220 & 436 & -4.22 \\
\hline 3 & 220 & 205 & 262 & 3 & 223 & 226 & 228 & 3 & 222 & 387 & 367 & 3 & 220 & 437 & 402 & 3 & 219 & 169 & 170 \\
\hline 3 & $\therefore 12$ & $\Leftrightarrow 27$ & .21 & 3 & 216 & 229 & 216 & 3 & $2-25$ & 167 & 175 & 3 & $2-26$ & 779 & -760 & 3 & $2-28$ & 550 & -5.35 \\
\hline 3 & $2-3 n$ & $2+8$ & -213 & 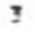 & $2-36$ & 127 & 116 & 3 & $2-37$ & 129 & -151 & 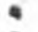 & $2-36$ & 528 & $55^{\circ}$ & * & $2-30$ & 171 & 176 \\
\hline • & $2-20$ & 168 & 172 & $\bullet$ & $2-27$ & 234 & 203 & 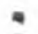 & $2-25$ & 300 & 305 & 5 & $3-36$ & 233 & -206 & 5 & $3-33$ & 152 & $-1 \geq 0$ \\
\hline 5 & $3-32$ & $1 \in 3$ & -111 & 5 & $3-27$ & 274 & -283 & 5 & $3-25$ & 250 & -253 & 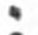 & $3-26$ & 297 & 333 & * & $3-20$ & 270 & 261 \\
\hline 4 & $3-33$ & $z t=$ & -238 & 3 & $\begin{array}{ll}317 \\
\end{array}$ & $=16$ & -536 & 3 & 319 & 202 & 186 & 2 & 322 & 360 & 324 & 2 & 320 & 471 & 307 \\
\hline 2 & 313 & 419 & 460 & 2 & 317 & 4rs & -10 & 2 & 316 & 878 & 845 & 2 & $3-22$ & 700 & -655 & 2 & $3-24$ & 563 & -547 \\
\hline 2 & $3-25$ & $2 n 7$ & -125 & 2 & $3-28$ & 182 & -160 & 2 & $3-32$ & 158 & 171 & 2 & $3-36$ & 330 & 390 & 1 & $3-21$ & 600 & -567 \\
\hline 1 & $3-27$ & 260 & -236 & 1 & $3-10$ & 764 & $-77 a$ & 1 & $3-18$ & 323 & -326 & 1 & $3-17$ & 439 & 457 & 1 & 312 & 214 & -210 \\
\hline 1 & 310 & 469 & -474 & 1 & 315 & 125 & 133 & 1 & 316 & 235 & -194 & 0 & $313^{\circ}$ & 1073 & 065 & 1 & 417 & 616 & 670 \\
\hline 1 & 410 & 450 & .42 & 1 & 412 & 475 & 465 & 1 & $4-15$ & 208 & -255 & 1 & $4-16$ & 413 & -375 & 1 & $4-17$ & 996 & -960 \\
\hline 1 & $0-17$ & $7 \approx 3$ & -20.7 & 1 & $4-20$ & 717 & -683 & 2 & 416 & $\$ 70$ & 413 & 2 & - 18 & 392 & .05 & 2 & - 23 & 365 & 573 \\
\hline 3 & 427 & 250 & -305 & 3 & 425 & 321 & -371 & 3 & 420 & 220 & -240 & 3 & - 18 & 260 & -322 & 3 & - 17 & 253 & 253 \\
\hline 3 & - 16 & $4 \pi 1$ & -503 & 3 & 420 & 250 & -295 & - & $0-38$ & 178 & -186 & - & $4-20$ & 188 & -211 & : & $4-27$ & 204 & $\begin{array}{r}-253 \\
315\end{array}$ \\
\hline 4 & $4-z^{2}$ & 204 & -122 & - & $4-23$ & 209 & -331 & 4 & $4-21$ & 129 & -178 & 4 & $4-19$ & 203 & -271 & 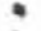 & 13 & 280 & $\begin{array}{r}315 \\
-370\end{array}$ \\
\hline$\Delta$ & 410 & 200 & -301 & $\bullet$ & 415 & 212 & 313 & * & 420 & 220 & -294 & $\bullet$ & $5-20$ & $3=1$ & -397 & * & $5-26$ & 288 & $\begin{array}{l}-326 \\
165\end{array}$ \\
\hline$\star$ & $5-20$ & 10.3 & $15^{\circ}$ & - & $5-33$ & 155 & 117 & 4 & $5-36$ & 152 & $13 *$ & 1 & $5-22$ & 147 & 186 & 1 & $5-21$ & 141 & \\
\hline 1 & $5-20$ & 209 & $28^{\circ}$ & 1 & $5-10$ & 431 & 460 & 1 & $5-17$ & 176 & 205 & 1 & $5-15$ & 451 & 532 & 1 & $5-13$ & $\begin{array}{l}333 \\
327\end{array}$ & $\begin{array}{r}633 \\
339\end{array}$ \\
\hline 1 & $s-11$ & 393 & 460 & 3 & $2-31$ & 152 & -164 & 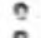 & 521 & 297 & 334 & ? & $\begin{array}{ll}520 \\
5\end{array}$ & $\begin{array}{l}489 \\
489\end{array}$ & 892 & $:$ & $\begin{array}{rr}5 & 19 \\
6-13\end{array}$ & $\begin{array}{l}327 \\
239\end{array}$ & $\begin{array}{l}339 \\
268\end{array}$ \\
\hline E & $\begin{array}{l}513 \\
4\end{array}$ & 644 & 605 & 0 & 516 & 500 & 532 & n & 515 & 196 & $\begin{array}{r}216 \\
-284\end{array}$ & 0 & $\begin{array}{ll}5 & 10 \\
6 & -9\end{array}$ & 296 & 300 & 0 & & 280 & -278 \\
\hline c & $\begin{array}{l}6-12 \\
6-7\end{array}$ & $\begin{array}{l}150 \\
106\end{array}$ & & e & $\begin{array}{l}6-11 \\
6-6\end{array}$ & $\begin{array}{l}337 \\
147\end{array}$ & $\begin{array}{r}353 \\
-175\end{array}$ & $:$ & $\begin{array}{l}6-10 \\
6-5\end{array}$ & $\begin{array}{l}237 \\
172\end{array}$ & $\begin{array}{r}284 \\
210\end{array}$ & 0 & $6=$ & 91 & -103 & 0 & $=-3$ & 196 & 252 \\
\hline i & $5-1$ & 130 & $\begin{array}{l}226 \\
132\end{array}$ & i & $\begin{array}{ll}6 & -6 \\
5 & 13\end{array}$ & 307 & -413 & 1 & 514 & 02 & 429 & 1 & 516 & 471 & +40 & 1 & 517 & 378 & -367 \\
\hline$i$ & 514 & 539 & $=70$ & 1 & 519 & 270 & 198 & 1 & 520 & 275 & 289 & 1 & 521 & 239 & 258 & 1 & 610 & 210 & -211 \\
\hline 1 & 60 & 152 & 101 & 1 & 67 & 255 & $2 \in 1$ & 1 & 65 & 292 & 288 & 1 & & 167 & 199 & 1 & & 277 & 315 \\
\hline 1 & $6-14$ & 269 & 268 & 1 & $6-17$ & 172 & -155 & 1 & $6-18$ & 350 & 366 & 1 & $6-20$ & 570 & 610 & 2 & $6-21$ & 216 & $\begin{array}{r}-131 \\
271\end{array}$ \\
\hline 1 & $6-22$ & $6 \mathrm{Cu}$ & $t 12$ & I & $6-23$ & 107 & -162 & 1 & $6-24$ & 493 & 498 & 1 & $6-25$ & 165 & -107 & 2 & $s-26$ & 239 & 271 \\
\hline 2 & 614 & 217 & -248 & 2 & 615 & 320 & 335 & 2 & 616 & 199 & -216 & 3 & 619 & 213 & 190 & 3 & 618 & 406 & 405 \\
\hline 3 & 612 & 374 & 382 & 3 & 614 & 277 & 281 & 4 & $s-4 n$ & 132 & 182 & $:$ & $6-38$ & 132 & $15 n$ & 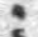 & $6-36$ & 163 & 227 \\
\hline 5 & $6-19$ & 139 & 98 & 5 & $6-22$ & 120 & 256 & 5 & $6-24$ & 216 & 246 & 5 & $6-26$ & 168 & 238 & 3 & $\begin{array}{l}6-28 \\
7-19\end{array}$ & 309 & $\begin{array}{r}216 \\
-352\end{array}$ \\
\hline 3 & $7-20$ & $1=9$ & 158 & 3 & $7-25$ & 212 & 190 & 3 & $\begin{array}{l}7-23 \\
7-12\end{array}$ & 199 & $\begin{array}{r}223 \\
-i m 9\end{array}$ & 3 & $\begin{array}{l}7-21 \\
7.13\end{array}$ & $\begin{array}{l}277 \\
226\end{array}$ & $\begin{array}{l}310 \\
235\end{array}$ & $i$ & 117 & 278 & 290 \\
\hline 1 & $\begin{array}{c}7-12 \\
7 \\
10\end{array}$ & 206 & $\begin{array}{l}167 \\
249\end{array}$ & $\frac{1}{2}$ & $\begin{array}{l}7-15 \\
8-25\end{array}$ & $\begin{array}{l}275 \\
101\end{array}$ & $\begin{array}{r}-334 \\
173\end{array}$ & $\frac{1}{2}$ & $\begin{array}{l}7-13 \\
8-23\end{array}$ & 247 & $2+9$ & i & 713 & 226 & 235 & & & & \\
\hline
\end{tabular}

